\title{
Detaching the antiferromagnetic quantum critical point from the Fermi-surface reconstruction in $\mathrm{YbRh}_{2} \mathrm{Si}_{2}$
}

\author{
S. Friedemann ${ }^{1 \star}$, T. Westerkamp ${ }^{1}$, M. Brando ${ }^{1}$, N. Oeschler ${ }^{1}$, S. Wirth ${ }^{1}$, P. Gegenwart ${ }^{1,2}$, C. Krellner ${ }^{1}$, \\ C. Geibel ${ }^{1}$ and F. Steglich ${ }^{1 \star}$
}

\begin{abstract}
A continuous phase transition driven to zero temperature by a non-thermal parameter, such as pressure, terminates in a quantum critical point (QCP). At present, two main theoretical approaches are available for antiferromagnetic QCPs in heavyfermion systems. The conventional one is the quantum generalization of finite-temperature phase transitions, which reproduces the physical properties in many cases ${ }^{1-5}$. More recent unconventional models incorporate a breakdown of the Kondo effect, giving rise to a Fermi-surface reconstruction ${ }^{6-8}-$ $\mathbf{Y b R h}_{2} \mathrm{Si}_{2}$ is a prototype of this category, ${ }^{5,9-11}$. In $\mathbf{Y b R h _ { 2 }} \mathbf{S i}_{2}$, the antiferromagnetic transition temperature merges with the Kondo breakdown at the QCP. Here, we study the evolution of the quantum criticality in $\mathrm{YbRh}_{2} \mathrm{Si}_{2}$ under chemical pressure. Surprisingly, for positive pressure we find the signature of the Kondo breakdown within the magnetically ordered phase, whereas negative pressure induces their separation, leaving an intermediate spin-liquid-type ground state over an extended range. This behaviour suggests a new quantum phase arising from the interplay of the Kondo breakdown and the antiferromagnetic QCP.
\end{abstract}

In heavy-fermion systems, the Kondo effect leads to the formation of composite quasiparticles of the $f$ and conductionelectron states with largely renormalized masses forming a Landau Fermi-liquid ground state in the paramagnetic regime well below the Kondo temperature $T_{\mathrm{K}}$. These quasiparticles are assumed to stay intact at the quantum critical point (QCP) in the conventional models in which magnetic order arises through a spin-densitywave (SDW) instability. However, the observation of magnetic correlations in $\mathrm{CeCu}_{5.9} \mathrm{Au}_{0.1}$ being of local character ${ }^{11}$ prompted a series of theoretical descriptions that discard this basic assumption. Rather, they focus on the breakdown of the Kondo effect, which causes the $f$ states to become localized and decoupled from the conduction-band states at the QCP where one expects the Fermi surface to be reconstructed ${ }^{7}$. Consequently, a new energy scale $T^{\star}$ is predicted reflecting the finite-temperature $T$ crossover of the Fermi-surface volume. This picture has been scrutinized in tetragonal $\mathrm{YbRh}_{2} \mathrm{Si}_{2}\left(T_{\mathrm{K}} \approx 25 \mathrm{~K}\right.$; ref. 12$)$, a stoichiometric and very clean heavy-fermion metal that seems to be ideally suited for this kind of study ${ }^{9,12}$ : antiferromagnetic order sets in at a very low temperature $T_{\mathrm{N}}=0.07 \mathrm{~K}$ and can easily be suppressed by a small magnetic field of $\mu_{0} H_{\mathrm{N}}=60 \mathrm{mT}$ ( $H \perp c$, with $c$ being the magnetically hard axis). Hall-effect experiments ${ }^{13}$ have detected a rapid change of the Hall coefficient along a line $T^{\star}(H)$ that converges with $H_{\mathrm{N}}$, the width of the Hall crossover extrapolating to zero for $T \rightarrow 0$. This change was considered evidence for an abrupt change of the Fermi-surface volume indicating a correspondence between $T^{\star}(H)$ and the Kondo-breakdown energy scale. Subsequent thermodynamic and transport investigations confirmed $T^{\star}(H)$ to be a new energy scale ${ }^{14}$. The a.c. susceptibility $\chi^{\prime}(T)$ turned out to exhibit a pronounced maximum at $T^{\star}$, which can be particularly well distinguished from the sharp signature at $T_{\mathrm{N}}$. The magnetoresistance exhibits a step-like crossover similar to the Hall coefficient. In fact, recent calculations for a Kondo lattice predict such a feature for both transport properties ${ }^{15}$. Furthermore, the magnetization shows a smeared kink at $T^{\star}(H)$ between two almost linear regimes with different slopes ${ }^{14}$. The anomaly at $T^{\star}(H)$ in all isothermal measurements can be described either by the same crossover function proposed for the Hall effect or its integral version resembling a smeared kink. The maximum in $\chi^{\prime}(T)$ is a natural consequence of the magnetization kink being smeared and shifted to higher fields as the temperature is raised.

The Fermi-surface reconstruction may also occur away from the antiferromagnetic QCP as observed in $\mathrm{CeIn}_{3}$ and $\mathrm{CeRh}_{1-x} \mathrm{Co}_{x} \mathrm{In}_{5}$ (refs 16, 17). It is therefore very important to understand the interplay between the phenomena assigned to the Kondo breakdown and the magnetic order. We address this issue by investigating $\mathrm{YbRh}_{2} \mathrm{Si}_{2}$ under positive and negative chemical pressure, which was realized by partial isoelectronic substitution of smaller Co or larger Ir for Rh, respectively (the analogy of chemical and external pressure is discussed in Supplementary Information $\mathrm{A}$ ). In $\mathrm{Yb}$ systems, pressure yields a stabilization of magnetism, in particular an increase of $T_{\mathrm{N}}$ (ref. 18). On the other hand, negative pressure, corresponding to a lattice expansion, reduces $T_{\mathrm{N}}$.

The $T-H$ phase diagrams of $\mathrm{Yb}\left(\mathrm{Rh}_{0.94} \mathrm{Ir}_{0.06}\right){ }_{2} \mathrm{Si}_{2}$ and $\mathrm{Yb}\left(\mathrm{Rh}_{0.93} \mathrm{Co}_{0.07}\right)_{2} \mathrm{Si}_{2}$ (labelled 6\% Ir and 7\% Co in the following) are compared with that of $\mathrm{YbRh}_{2} \mathrm{Si}_{2}$ in Fig. 1. This set emphasizes the evolution of the various energy scales. First, the magnetic one follows the expected pressure dependence: for $6 \% \mathrm{Ir}, T_{\mathrm{N}}$ is depressed below $0.02 \mathrm{~K}$, whereas in the case of $7 \% \mathrm{Co}, T_{\mathrm{N}}$ is enhanced to $0.41 \mathrm{~K}$. Second, the energy scale $T^{\star}(H)$ virtually does not change its position in the $T-H$ phase diagram. Consequently, $T^{\star}(H)$ is separated from $T_{\mathrm{N}}(H)$ in $6 \% \mathrm{Ir}$, whereas they intersect in $7 \%$ Co. Finally, at fields above the respective critical fields $H^{\star}$ (for $6 \% \mathrm{Ir}$ ) and $H_{\mathrm{N}}$ (for 7\% Co) at which $T^{\star}(H)$ and $T_{\mathrm{N}}(H)$ vanish, the Fermi-liquid phase forms below $T_{\mathrm{FL}}(H)$.

These findings were mainly deduced from the temperaturedependent a.c. susceptibility $\chi^{\prime}(T)$ shown in Fig. 2. For $6 \%$ Ir,

${ }^{1}$ Max-Planck-Institut für Chemische Physik fester Stoffe, D-01187 Dresden, Germany, ${ }^{2}$ I. Physik. Institut, Georg-August-Universität Göttingen, D-37077 Göttingen, Germany. ${ }^{*}$-mail: Sven.Friedemann@cpfs.mpg.de; Steglich@cpfs.mpg.de. 


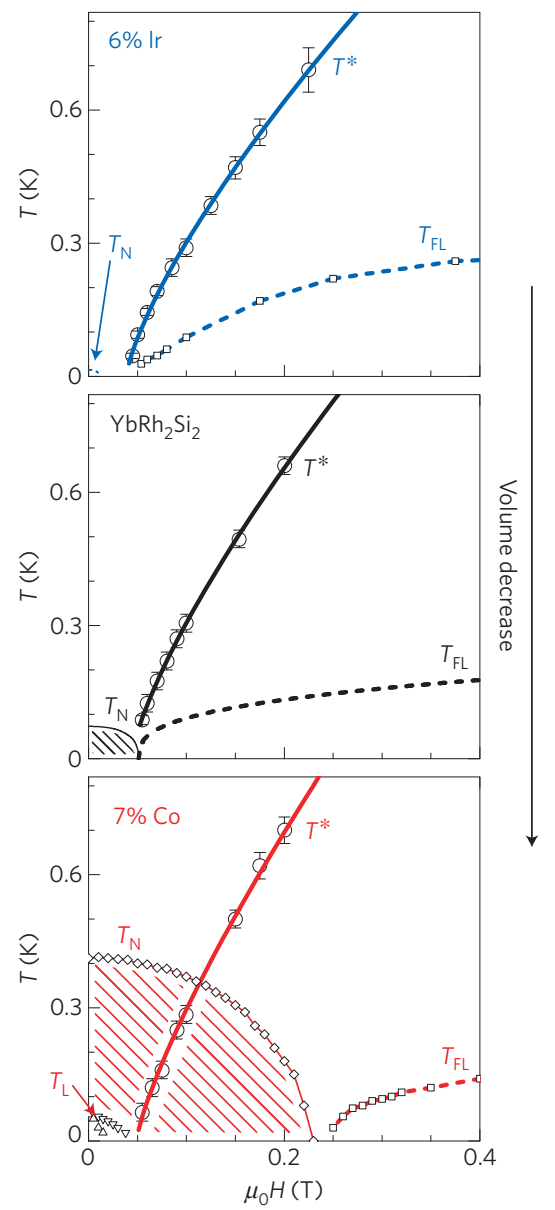

Figure 1 | Evolution of the $\mathrm{T}-\mathrm{H}$ phase diagram of $\mathrm{YbRh}_{2} \mathrm{Si}_{2}$ under negative and positive chemical pressure. The open circles represent the position of $T^{\star}(H)$ determined from the maximum of the a.c. susceptibility $\chi^{\prime}(T)$. The crossover temperature to the Landau Fermi-liquid state, $T_{\mathrm{FL}}$, was deduced from the onset of the quadratic temperature dependence in the electrical resistivity $\rho(T)$. In the phase diagram of $\mathrm{Yb}\left(\mathrm{Rh}_{0.94} \mathrm{Ir}_{0.06}\right)_{2} \mathrm{Si}_{2}$ (upper panel), the dotted line renders the phase boundary of the antiferromagnetically ordered state estimated from that of $\mathrm{YbRh}_{2} \mathrm{Si}_{2}$ by scaling both axes with $a=T_{N} 6 \% \mathrm{Ir} / T_{\mathrm{N}} \mathrm{YbRh}_{2} \mathrm{Si}_{2} \approx 0.29$ (see Supplementary Information A). For $\mathrm{Yb}\left(\mathrm{Rh}_{0.93} \mathrm{Co}_{0.07}\right)_{2} \mathrm{Si}_{2}$ (lower panel), the Néel-temperature $T_{\mathrm{N}}$ was determined from $\rho(T)$. The lower transition at $T_{\mathrm{L}}$ as derived from $\chi^{\prime}$ bifurcates in finite fields (see Supplementary Information B). The data for pure $\mathrm{YbRh}_{2} \mathrm{Si}_{2}$ are reproduced from ref. 14. The lines are guides to the eye. The error bars represent standard errors.

no signature of magnetic order is observed as the zero-field curve increases monotonically with decreasing $T$. An antiferromagnetically ordered state is anticipated at even lower temperatures (see Supplementary Information A). On the other hand, $\chi^{\prime}(T)$ of $7 \%$ Co exhibits a sharp kink at $T_{\mathrm{N}}=0.41 \mathrm{~K}$ and a cusp at $T_{\mathrm{L}}=0.06 \mathrm{~K}$, the critical temperature of a second, presumably also antiferromagnetic, transition. In external fields, these two transitions are shifted to lower temperatures, with the lower one bifurcating as detailed in Supplementary Information B. For $6 \%$ Ir and 7\% Co, a maximum in $\chi^{\prime}(T)$ assigned to $T^{\star}(H)$ is observed above $45 \mathrm{mT}$ and $55 \mathrm{mT}$, respectively. In an increasing field, this maximum shifts to higher temperatures. Remarkably, for $7 \%$ Co the maximum appears both below $T_{\mathrm{N}}$ at small fields, and above $T_{\mathrm{N}}$ at fields of $150 \mathrm{mT}$ and higher. This clearly illustrates that the energy scales, $T_{\mathrm{N}}(H)$ and $T^{\star}(H)$, indeed intersect.

In Fig. 3, we compare the signatures of $T^{\star}(H)$ in magnetoresistance as well as in magnetization for stoichiometric $\mathrm{YbRh}_{2} \mathrm{Si}_{2}$,
$7 \%$ Co and $6 \%$ Ir. At $0.5 \mathrm{~K}$, the magnetoresistance curves of these three samples exhibit an almost identical, step-like crossover. In particular, the inflection point assigned to $T^{\star}(H)$ (ref. 14) is nearly unchanged by chemical pressure, resembling the results from susceptibility and magnetization (discussed below). In the case of $7 \%$ Co, this holds true above $T_{\mathrm{N}}$, whereas at lower temperatures the inflection point is locked to the antiferromagnetic phase boundary (not shown).

We analysed the magnetization $M$ as outlined in supporting online material for ref. 14 as $\tilde{M}=M+\partial M / \partial H \cdot H=M+\chi^{\prime} H$. In Fig. 3b, we focus on the data at the lowest temperatures, which unambiguously prove the existence of the $T^{\star}$ anomaly also within the antiferromagnetic phase of $7 \%$ Co. $\tilde{M}(H)$ exhibits a broadened kink at $T^{\star}(H)$ between two linear regimes with different slopes $^{14}$. In addition to, but clearly distinct from, this kink at $T^{\star}(H)$, a small peak is observed at $220 \mathrm{mT}$ for $7 \% \mathrm{Co}$, which is related to the critical field of the ordered phase. Therefore, our magnetization and susceptibility results on $7 \%$ Co yield striking evidence for the crossover at $T^{\star}(H)$ to also occur inside the antiferromagnetic ordered phase. A re-examination of existing magnetization data ${ }^{19}$ confirms this finding for $\mathrm{YbRh}_{2} \mathrm{Si}_{2}$ under external pressure, supporting the equivalence to chemical pressure. To check for possible disorder effects, a comprehensive study of $\mathrm{YbRh}_{2} \mathrm{Si}_{2}$ under hydrostatic pressure is in preparation.

Exactly such an intersection of $T_{\mathrm{N}}(H)$ and $T^{\star}(H)$, as observed for $7 \% \mathrm{Co}$, is expected in the three-dimensional (3D) SDW theory. As shown in Supplementary Information D, the field dependence of the Néel temperature in the vicinity of $H_{\mathrm{N}}$ indeed follows the anticipation of the 3D SDW theory. However, ref. 6 predicts that critical fluctuations ought to be $2 \mathrm{D}$ once $T_{\mathrm{N}}(H)$ and $T^{\star}(H)$ converge at the antiferromagnetic QCP, as observed for pure $\mathrm{YbRh}_{2} \mathrm{Si}_{2}$ (refs 13, 14).

We now turn to the interesting case of $6 \%$ Ir where, according to our results, the critical fields $H_{\mathrm{N}}$ and $H^{\star}$ seem to become separated from each other (Fig. 1, top panel). The resistivity versus temperature curves measured in various fields are shown in Fig. 4. In zero field, $\rho(T)$ is quasi-linear below $1 \mathrm{~K}$ with a slight upward curvature at the lowest temperatures. In small external fields, this curvature is reduced, yielding the steepest curve at $50 \mathrm{mT}$. A $T^{2}$ form indicative of a Fermi-liquid ground state is observed only at fields exceeding $50 \mathrm{mT}$ (see the lines in Fig. 4). The $A$ coefficient in the Fermi-liquid regime, which is proportional to the effective quasiparticle-quasiparticle scattering cross-section, follows a $\left(H-H_{\mathrm{c}}^{A}\right)^{-1}$ divergence with a critical field of $\mu_{0} H_{\mathrm{c}}^{A}=30$ (5) mT (Fig. 4, bottom inset) close to $H_{\mathrm{FL}}=35(5) \mathrm{mT}$, the field at which $T_{\mathrm{FL}}$ vanishes in the zero temperature limit. An important finding of this study is that $H_{\mathrm{FL}}$ is substantially larger than the critical field of the antiferromagnetically ordered phase $\mu_{0} H_{\mathrm{N}} \approx 15 \mathrm{mT}$. In addition, the $T^{\star}(H)$ line extrapolates to a critical field $\mu_{0} H^{\star} \approx 40 \mathrm{mT}$ in close vicinity to $H_{\mathrm{FL}}$ and $H_{\mathrm{c}}^{A}$ (see Fig. 4 , top inset). Consequently, our results reveal a finite field range within which the resistivity exhibits non-Fermi-liquid behaviour. Obviously, neither the crossover at $T^{\star}(H)$ nor that at $T_{\mathrm{FL}}(H)$ is linked to the critical field of the antiferromagnetically ordered phase. We note that similar behaviour was observed for $\mathrm{Yb}_{0.95} \mathrm{La}_{0.05} \mathrm{Rh}_{2} \mathrm{Si}_{2}$, for which $\rho(T)$ is linear in fields up to $40 \mathrm{mT}$, where neither a magnetically ordered nor a Fermi-liquid ground state was found ${ }^{20}$.

The divergence of the $A$ coefficient backs the presence of a QCP connected with the vanishing energy scale $T^{\star}$. Further support for a QCP at $H^{\star}$ stems from the analysis of the resistivity exponent $n$ in $\rho(T)-\rho_{0} \propto T^{n}$, shown in Fig. 4, top inset, as a coloured contour plot. The blue region $(n=2)$ reflects Fermi-liquid behaviour. Deviations are ascribed to the quantum criticality ${ }^{9}$ : in fact, the red region $(n=1)$ is clearly linked to $T^{\star}$ and well separated from the critical field of the antiferromagnetic order. 

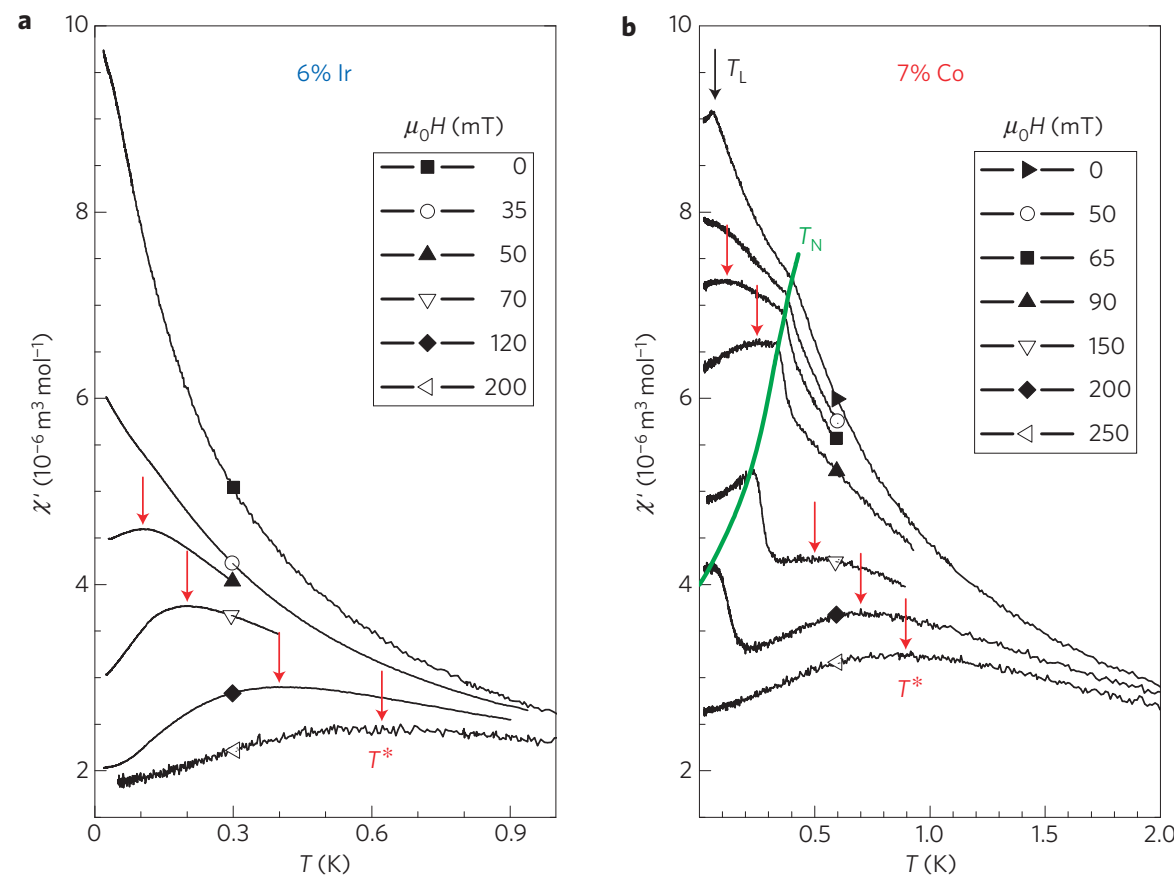

Figure 2 | Signatures of the energy scales $\boldsymbol{T}^{\star}(\boldsymbol{H})$ and $\boldsymbol{T}_{\mathbf{N}}(\boldsymbol{H})$ in the susceptibility. a,b, Temperature-dependent a.c. susceptibility $\chi^{\prime}(T)$ of $\mathrm{Yb}\left(\mathrm{Rh}_{0.94} \mathrm{Ir}_{0.06}\right)_{2} \mathrm{Si}_{2}(\mathbf{a})$ and $\mathrm{Yb}\left(\mathrm{Rh}_{0.93} \mathrm{Co}_{0.07}\right)_{2} \mathrm{Si}_{2}(\mathbf{b})$ at selected magnetic fields $H \perp c$. The red arrows indicate the temperatures of the maxima assigned to $T^{\star}$. For the 7\% Co sample, the onset of antiferromagnetic ordering in $\chi^{\prime}(T)$ is marked by the green line. A second transition at $T_{\mathrm{L}}$ is reflected as a peak in $\chi^{\prime}(T)$, marked by a black arrow.
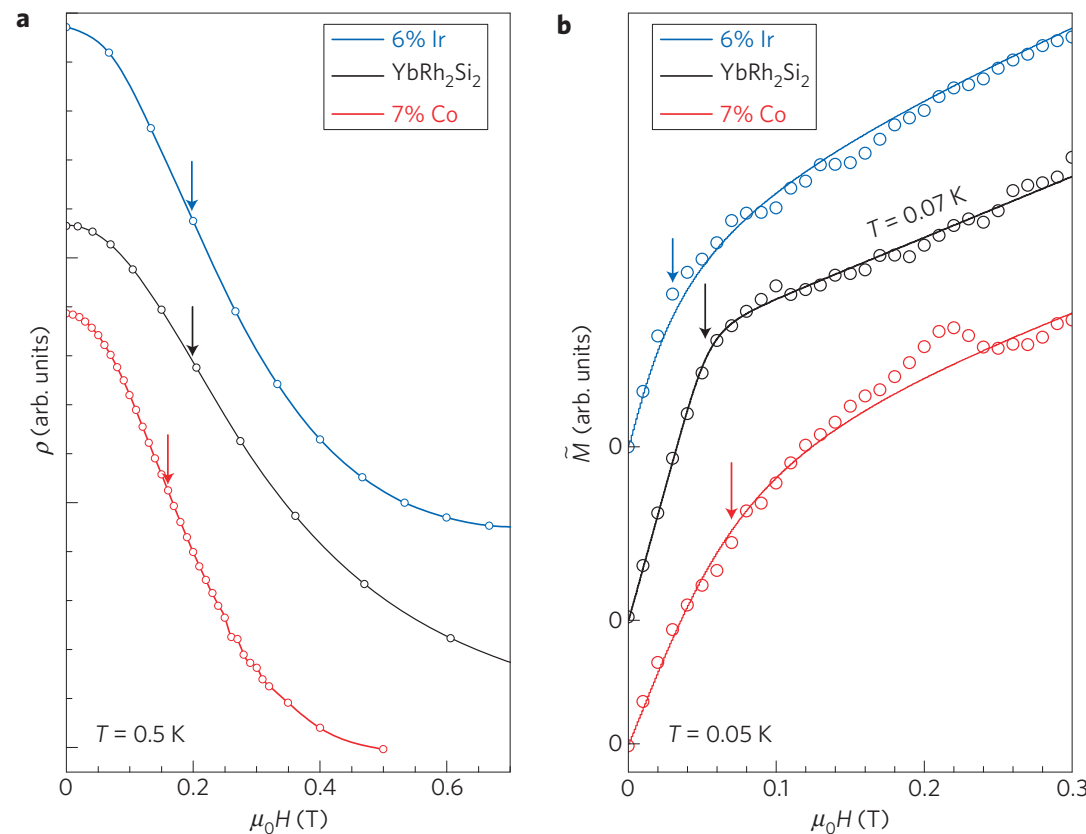

Figure 3 | Signatures of $\boldsymbol{T}^{\star}(\boldsymbol{H})$ in magnetoresistance and magnetization. a,b, Field dependence of magnetoresistance for $\mathrm{Yb}_{\mathrm{b}}\left(\mathrm{Rh}_{0.94} \mathrm{Ir}_{0.06}\right)_{2} \mathrm{Si}_{2}$, $\mathrm{Yb}\left(\mathrm{Rh}_{0.93} \mathrm{Co}_{0.07}\right)_{2} \mathrm{Si}_{2}$ and $\mathrm{YbRh}_{2} \mathrm{Si}_{2}$ at $0.5 \mathrm{~K}(\mathbf{a})$ and $\tilde{M}=M+\chi^{\prime} \mathrm{H}(\mathbf{b})$. Whereas $\tilde{M}$ curves for the former two samples are taken at $0.05 \mathrm{~K}$, the one for $\mathrm{YbRh}_{2} \mathrm{Si}_{2}$ is at $T_{\mathrm{N}}=0.07 \mathrm{~K}$ to avoid interference of $T^{\star}$ and $T_{\mathrm{N}}$. The lines in $\mathbf{b}$ represent fits of equation (1) to the data. The arrows in $\mathbf{a}$ and $\mathbf{b}$ mark the inflection point and the crossover field $H_{0}$, respectively (see the Methods section for the fitting procedure used to extract $H_{0}$ ). Both features are assigned to $T^{\star}(H)$ (see Supplementary Information C).

To underline that the QCP at $H^{\star}$ cannot be of magnetic origin, Supplementary Information $\mathrm{E}$ shows the $T^{\star}(H)$ line to be still at the same position in the phase diagram for $17 \%$ Ir substitution, although here no antiferromagnetic order is expected at all ${ }^{21}$. This indicates a separation of the antiferromagnetic QCP from the Kondo-breakdown QCP. In the intermediate field range, the local $f$ moments are expected to be neither Kondo screened nor antiferromagnetically ordered. This highlights a new metallic 'spin-liquid'-type ground state that has to be explored in more detail. The existence of a 'spin liquid' in a Kondo lattice has been speculated $^{8,22}$, but the conditions under which it might be realized remain uncertain. The quasi-linear resistivity predicted in 


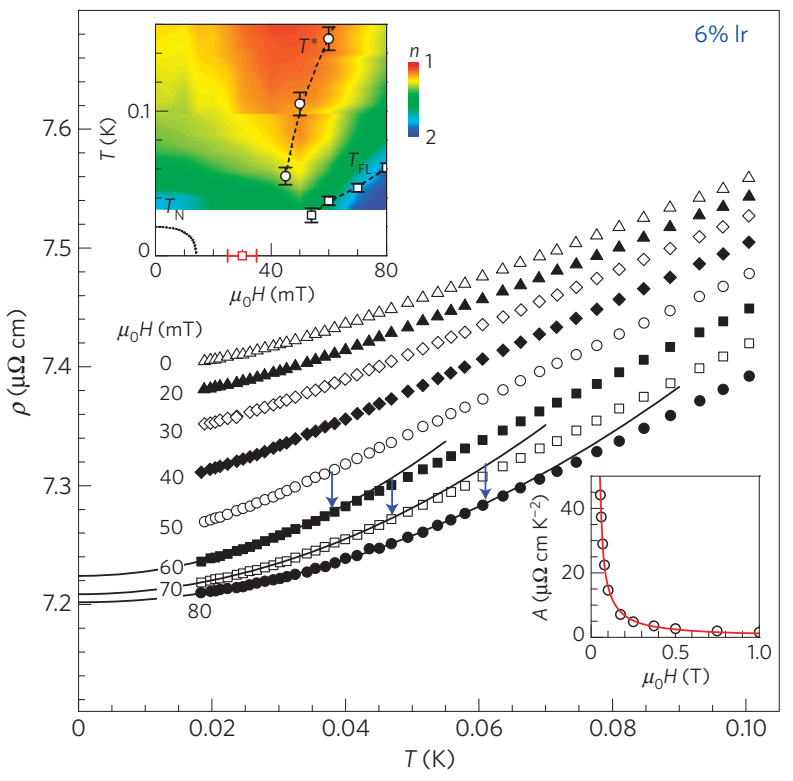

Figure 4 | Signatures of Fermi-liquid and non-Fermi-liquid behaviour in the resistivity of $\mathbf{Y b}\left(\mathbf{R h}_{\mathbf{0 . 9 4}} \mathbf{I r}_{\mathbf{0 . 0 6}}\right)_{\mathbf{2}} \mathbf{S i}_{\mathbf{2}}$. Temperature dependence of the resistivity $\rho(T)$ at selected magnetic fields. The lines indicate Fermi-liquid behaviour, that is, fits to $\rho(T)=\rho_{0}+A T^{n}$ with the exponent $n=2$ and $\rho_{0}$ being the residual resistivity, for temperatures below $T_{\mathrm{FL}}$ (marked by blue arrows). Bottom inset: The field dependence of the coefficient $A=\left(\rho-\rho_{0}\right) / T^{n}$ in the Fermi-liquid regime. The red line corresponds to $A(H) \propto\left(H-H_{c}^{A}\right)^{-1}$ yielding a critical field of $\mu_{0} H_{c}^{A}=30(5) \mathrm{mT}$. Top inset: Colour-coded representation of the resistivity exponent calculated as $n=\operatorname{dlog}\left(\rho-\rho_{0}\right) / \operatorname{dlog} T$. The energy scales $T_{N}, T^{\star}$ and $T_{\mathrm{FL}}$ are reproduced from Fig. 1, top panel. The red square on the abscissa depicts $H_{c}^{A}$, the critical field of the divergence of the $A$ coefficient (see bottom inset). The error bars represent standard errors.

ref. 22 resembles our experimental observations. Also for YbAgGe, a finite field range was reported where the resistivity exhibits similar non-Fermi-liquid behaviour to the lowest temperatures ${ }^{23}$. However, the specific heat of YbAgGe shows a saturation of $C(T) / T$ in this field range, discarding a spin-liquid ground state ${ }^{24}$. In contrast, preliminary measurements on $6 \%$ Ir down to $0.06 \mathrm{~K}$ reveal a strong divergence of $C(T) / T$ with decreasing temperature in the field range below $50 \mathrm{mT}$ (not shown), supporting our claim of a spin liquid. In addition, the susceptibility continues to increase towards the lowest temperatures (see Fig. 2a). The experimental evidence of such a new, non-magnetic ground state is fascinating and will certainly motivate future experimental and theoretical studies.

Figure 5 shows the evolution of the two different QCPs as a function of Ir/Co substitution. The following main results can be deduced from this figure. (1) The antiferromagnetic state is stabilized through the application of positive chemical pressure, as expected. (2) The position of the suggested breakdown of the Kondo effect depends only weakly on chemical pressurealthough the Kondo effect itself is known to be strongly pressure dependent. (3) As a consequence, for positive pressure, the antiferromagnetic QCP at $H_{\mathrm{N}}$ is located in the regime with intact Kondo screening $\left(H_{\mathrm{N}}>H^{\star}\right)$ where the SDW theory is expected to be applicable in accordance with our observations. (4) For negative chemical pressure, on the other hand, $H_{\mathrm{N}}$ is separated from $H^{\star}$ towards lower fields with an intermediate spin-liquid-type ground state emerging. Obviously, here, antiferromagnetic order and the Fermi-liquid ground state are not connected by a single QCP, but are separated by a spin liquid, that is, a non-Fermi-liquid range as previously observed for $\mathrm{MnSi}$ (ref. 25) and, perhaps, in $\beta$ - $\mathrm{YbAlB}_{4}$ (refs 26, 27).

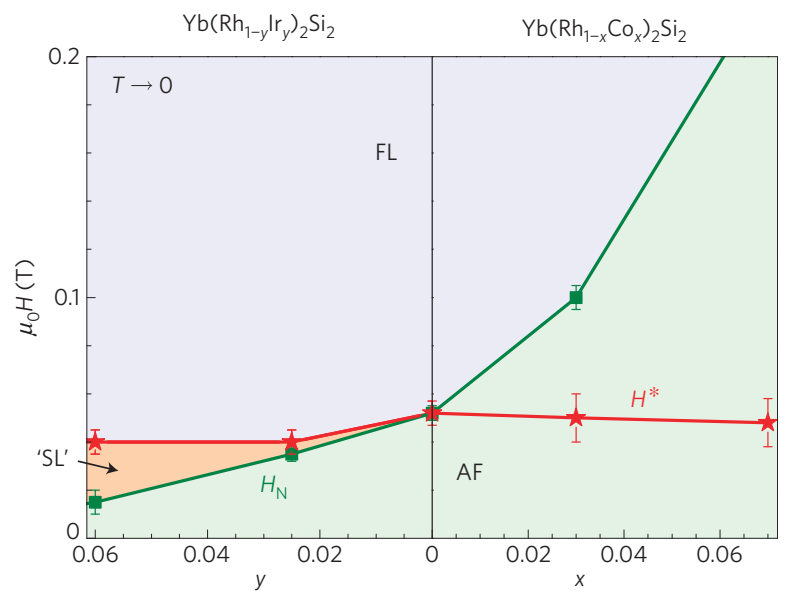

Figure 5 | Experimental phase diagram in the zero temperature limit. The zero-temperature phase diagram depicts the extrapolated critical fields of the various energy scales. The red line represents the critical field of $T^{\star}(H)$ and the green line the antiferromagnetic (AF) critical field $H_{N}$. The blue and green regions mark the Fermi-liquid (FL) and magnetically ordered ground state, respectively. The orange region situated between these two is a phase that resembles a spin liquid (SL). Data for $\mathrm{Yb}\left(\mathrm{Rh}_{1-x} \mathrm{M}_{x}\right)_{2} \mathrm{Si}_{2}$ with 2.5\% Ir and 3\% Co have been included. The error bars represent standard errors.

To conclude, the application of chemical pressure provides a wider view on the global phase diagram of $\mathrm{YbRh}_{2} \mathrm{Si}_{2}$ by lifting the coincidence of the multiple energy scales in the stoichiometric compound. The results and their interpretation presented here pose a formidable challenge for those theories describing the breakdown of the Kondo effect near an antiferromagnetic QCP in Kondo lattice systems. It remains to be explored under which conditions antiferromagnetic ordering and the Fermi-surface reconstruction may eventually become separated as observed for $\mathrm{YbRh}_{2} \mathrm{Si}_{2}$ with Ir substitution. Equally important, it needs to be understood why in pure $\mathrm{YbRh}_{2} \mathrm{Si}_{2}$, the antiferromagnetic QCP coincides with the Kondo breakdown.

\section{Methods}

Single crystals were grown from In flux, analogous to the stoichiometric samples described earlier ${ }^{12}$. The In flux was subsequently removed in hydrochloric acid. The presented results prove the absence of residual In. X-ray diffraction confirms the single crystallinity. All low-temperature measurements were carried out with the magnetic field aligned perpendicular to the crystallographic $c$ axis, $H \perp c$. The a.c.-susceptibility measurements were carried out at low frequencies with a modulation field amplitude of $4 \mu \mathrm{T}$ down to $0.02 \mathrm{~K}$. As no imaginary signal was detected, the real part $\chi^{\prime}$ is a direct measure of the field derivative of the magnetization. The temperature-dependent susceptibility $\chi^{\prime}(T)$ was measured in selected static magnetic fields. The isothermal susceptibility $\chi^{\prime}(H)$ was measured as a function of a field applied in addition to the modulation field. The electrical resistivity $\rho$ was monitored by a standard four-point lock-in technique at low frequencies down to $0.02 \mathrm{~K}$. An extremely small out-of-phase signal of less than $1 \%$ proves the high quality of the spot-welded contacts. With the help of low-temperature transformers, a very high sensitivity of better than $0.1 \mathrm{nV}$ was realized. In all samples, the resistivity was measured perpendicular to the crystallographic $c$ axis, and the magnetic field was applied parallel to the current. The magnetic field dependence of the magnetization $M(H)$ was isothermally measured in a high-resolution Faraday magnetometer down to $0.05 \mathrm{~K}$ (ref. 28). Background contributions from the sample platform and the torque exerted on the sample have been subtracted. The magnetization was analysed in the form $\tilde{M}=M+\chi^{\prime} H$ by fitting

$$
\int_{0}^{H} A_{2}-\left(A_{2}-A_{1}\right) /\left(1+\left(H^{\prime} / H_{0}\right)^{p}\right) \mathrm{d} H^{\prime}
$$

to the data from which the crossover field $H_{0}$ was obtained ${ }^{14} \cdot \tilde{M}$ is preferred for the analysis as it enables a more precise fitting compared with $M$ itself, although the conclusions drawn from $M(H)$ are identical (see ref. 14 and its supporting online material). 
Received 7 January 2009; accepted 28 April 2009; published online 31 May 2009

\section{References}

1. Hertz, J. A. Quantum critical phenomena. Phys. Rev. B 14, 1165-1184 (1976).

2. Millis, A. J. Effect of a nonzero temperature on quantum critical-points in itinerant fermion systems. Phys. Rev. B 48, 7183-7196 (1993).

3. Moriya, T. \& Takimoto, T. Anomalous properties around magnetic instability in heavy electron systems. J. Phys. Soc. Jpn. 64, 960-969 (1995).

4. Löhneysen, H. v., Rosch, A., Vojta, M. \& Wölfle, P. Fermi-liquid instabilities at magnetic quantum phase transitions. Rev. Mod. Phys. 79, 1015-1075 (2007).

5. Gegenwart, P., Si, Q. \& Steglich, F. Quantum criticality in heavy-fermion metals. Nature Phys. 4, 186-197 (2008).

6. Si, Q., Rabello, M. S., Ingersent, K. \& Smith, J. L. Locally critical quantum phase transitions in strongly correlated metals. Nature 413, 804-808 (2001).

7. Coleman, P., Pépin, C., Si, Q. \& Ramazashvili, R. How do Fermi liquids get heavy and die? J. Phys. Condens. Matter 13, R723-R738 (2001).

8. Senthil, T., Vojta, M. \& Sachdev, S. Weak magnetism and non-Fermi liquids near heavy-fermion critical points. Phys. Rev. B 69, 035111 (2004).

9. Custers, J. et al. The break-up of heavy electrons at a quantum critical point Nature 424, 524-527 (2003).

10. Park, T. et al. Isotropic quantum scattering and unconventional superconductivity. Nature 456, 366-368 (2008).

11. Schröder, A. et al. Onset of antiferromagnetism in heavy-fermion metals. Nature 407, 351-355 (2000).

12. Trovarelli, O. et al. $\mathrm{YbRh}_{2} \mathrm{Si}_{2}$ : Pronounced non-Fermi-liquid effects above a low-lying magnetic phase transition. Phys. Rev. Lett. 85, 626-629 (2000).

13. Paschen, S. et al. Hall-effect evolution across a heavy-fermion quantum critical point. Nature 432, 881-885 (2004).

14. Gegenwart, P. et al. Multiple energy scales at a quantum critical point. Science 315, 969-971 (2007).

15. Coleman, P., Marston, J. B. \& Schofield, A. J. Transport anomalies in a simplified model for a heavy-electron quantum critical point. Phys. Rev. B 72, 245111 (2005)

16. Harrison, N. et al. Fermi surface of $\mathrm{CeIn}_{3}$ above the Néel critical field. Phys. Rev. Lett. 99, 056401 (2007).

17. Goh, S. K. et al. Fermi-surface reconstruction in $\mathrm{CeRh}_{1-x} \mathrm{Co}_{x} \mathrm{In}_{5}$. Phys. Rev. Lett. 101, 056402 (2008)

18. Goltsev, A. V. \& Abd-Elmeguid, M. M. Origin of the pressure dependence of the Kondo temperature in Ce- and Yb-based heavy-fermion compounds. J. Phys. Condens. Matter 17, S813-S821 (2005).
19. Tokiwa, Y. et al. Field-induced suppression of the heavy-fermion state in $\mathrm{YbRh}_{2} \mathrm{Si}_{2}$. Phys. Rev. Lett. 94, 226402 (2005).

20. Weickert, F., Gegenwart, P., Ferstl, J., Geibel, C. \& Steglich, F. Low-temperature electrical resistivity of $\mathrm{Yb}_{1-x} \mathrm{La}_{x} \mathrm{Rh}_{2} \mathrm{Si}_{2}$. Physica B 378-380, 72-73 (2006).

21. Westerkamp, T., Gegenwart, P., Krellner, C., Geibel, C. \& Steglich, F. Low-temperature magnetic susceptibility of $\mathrm{Yb}\left(\mathrm{Rh}_{1-x} \mathrm{M}_{x}\right)_{2} \mathrm{Si}_{2}(\mathrm{M}=\mathrm{Ir}, \mathrm{Co})$ single crystals. Physica B 403, 1236-1238 (2008).

22. Pépin, C. Selective Mott transition and heavy fermions. Phys. Rev. B 77, 245129 (2008)

23. Niklowitz, P. G., Knebel, G., Flouquet, J., Bud'ko, S. L. \& Canfield, P. C. Field-induced non-Fermi-liquid resistivity of stoichiometric $\mathrm{YbAgGe}$ single crystals. Phys. Rev. B 73, 125101 (2006).

24. Tokiwa, Y. et al. Low-temperature thermodynamic properties of the heavy-fermion compound $\mathrm{YbAgGe}$ close to the field-induced quantum critical point. Phys. Rev. B 73, 094435 (2006).

25. Doiron-Leyraud, N. et al. Fermi-liquid breakdown in the paramagnetic phase of a pure metal. Nature 425, 595-599 (2003).

26. Nakatsuji, S. et al. Superconductivity and quantum criticality in the heavy-fermion system $\beta$-YbAlB 4 . Nature Phys. 4, 603-607 (2008).

27. Nevidomskyy, A. H. \& Coleman, P. Layered Kondo lattice model for quantum critical $\beta$-YbAlB 4 . Phys. Rev. Lett. 102, 077202 (2009)

28. Sakakibara, T., Mitamura, H., Tayama, T. \& Amitsuka, H. Faraday force magnetometer for high-sensitivity magnetization measurements at very low temperatures and high fields. Jpn. J. Appl. Phys. 33, 5067-5072 (1994).

\section{Acknowledgements}

The authors would like to thank P. Coleman and Q. Si for motivating discussions. We acknowledge partial support by the DFG Research Group 960 'Quantum Phase Transitions'.

\section{Author contributions}

S.F. set up, carried out and analysed the resistivity measurements. T.W. set up, carried out and analysed the a.c.-susceptibility measurements. M.B. set up, carried out and analysed the magnetization measurements. C.K. and C.G. grew the single crystals for the study. F.S., P.G., S.W. and N.O. planned and headed the project. S.F. wrote the paper with assistance from F.S., N.O, M.B., P.G. and S.W.

\section{Additional information}

Supplementary information accompanies this paper on www.nature.com/naturephysics. Reprints and permissions information is available online at http://npg.nature.com/ reprintsandpermissions. Correspondence and requests for materials should be addressed to S.F. or F.S. 